\title{
MAGMA SOLITONS
}

\author{
David R. Scott \\ Seismological Laboratory, California Institute of Technology
}

David J. Stevenson

Division of Geological and Planetary Science, California Institute of Technology

Abstract. Motivated to understand the process of melt migration in the earth's mantle, we have studied a generalised form of Darcy's law that describes porous flow in a matrix that can deform by creep. We find a remarkable richness of phenomena, including a new class of solitons. These consist of shape-preserving waves of high liquid fraction which buoyantly ascend through a stationary matrix. This may have important implications for the morphology and geochemistry of primary igneous processes, and applicability to other porous flow problems.

\section{Formulation}

Porous flow processes [Bear, 1972] occur in many natural systems. They allow, for example, the movement of water or hydrocarbons through sedimentary rocks and the movement of water through glacial ice; our interest lies in the migration of melt through partially molten rocks in a planetary interior [Turcotte, 1982]. The governing equation, known as Darcy's law, states that the flux of liquid through a permeable matrix is proportional to the pressure gradient in the liquid. This equation is usually stated in simple algebraic form but must be generalised to a non-linear partial differential equation if the matrix can deform by creep. This deformation takes the form of compaction or distension and is a response to spatial variations of liquid content.

Although the phenomena described here are novel, the basic equations have been obtained independently by several workers [Sleep, 1974; Stevenson, 1980; Fowler, 1984; McKenzie, 1984], with minor differences. A conceptual development is given here. Consider a partially molten medium consisting of a solid matrix phase and a liquid phase, both fully connected and incompressible, in a uniform vertical gravitational field. For clarity in this description, we neglect phase transitions and allow only vertical motions. Conservation of mass and energy in an elemental layer can be stated as:

$$
\begin{gathered}
\frac{\partial}{\partial z}\left(f v_{1}\right)=-\frac{\partial}{\partial z}\left[(1-f) v_{m}\right]=-\frac{\partial f}{\partial t} \\
\frac{\partial}{\partial z}\left[u\left(\sigma_{1}-\sigma_{m}\right)\right]+g \Delta p u=\frac{\eta_{1}}{k} u^{2}+\left(\sigma_{1}-\sigma_{m}\right) \frac{\partial u}{\partial z}
\end{gathered}
$$

where $z$ is the vertical coordinate, $t$ is time, $f$ is the mean volume fraction of liquid, $v_{1}$ and $v_{m}$ are the mean liquid and matrix velocities, $u=f v_{l}=-(1-f) v_{m}$ is the mean liquid flux in the barocentric frame, $\sigma_{1}$ and $\sigma_{\mathrm{m}}$ are the mean vertical nor-

Copyright 1984 by the American Geophysical Union.

Paper number 4L6341.

0094-8276/84/004L-6341\$03.00 mal stresses in the liquid and matrix, $\Delta \rho$ is the density difference between matrix and liquid phases (positive when the liquid is buoyant), $\mathrm{g}$ is the gravitational acceleration, $\eta_{1}$ is the liquid viscosity, and $\mathrm{k}$ is the matrix permeability.

Equation (1) is the continuity condition (neglecting $\Delta \rho$ ). The physical meanings of the terms in equation (2) are as follows. On the LHS, the first term is the divergence of the power into the layer per unit area (arising from the different stress states in the matrix and liquid when the matrix is deforming), and the second term is gravitational energy released per unit volume. On the RHS, the first term is the viscous dissipation in the liquid, which on general principles must scale as the liquid viscosity and as the square of the mean liquid flux. The factor of $1 / \mathrm{k}$ guarantees that the equation reduces to the algebraic form of Darcy's law when $\sigma_{1}=\sigma_{\mathrm{m}}$. The second term on the RHS is the appropriate form for the viscous dissipation in the matrix, assuming a linear rheology, since $\sigma_{l}-\sigma_{\mathrm{m}}$ is the stress difference driving the volumetric strain, $\partial u / \partial z$. To ensure that this dissipation is positive definite, we must assume a constitutive equation of the form $\sigma_{1}-\sigma_{m}=\eta^{*} \partial u / \partial z$. This equation defines $\eta^{*}$, an effective viscosity characterising the rate of matrix compaction or distension. This constitutive form has been obtained rigorously for some idealised models of the microscopic structure of the matrix. For example, if the liquid migrates along uniform cylindrical vertical conduits, analogous to glacier boreholes [Nye, 1953; Fowler, 1984], we find $\eta^{*} \approx \eta_{\mathrm{m}} / \mathrm{f}$, where $\eta_{m}$ is the shear viscosity of matrix phase (without porosity). In this case $\eta^{*}$ becomes large at small liquid fractions.

In the limit $f \ll<$, equations (1) and (2) take the following non-dimensional form:

$$
\begin{gathered}
u_{z}=-f_{t} \\
u=f^{n}\left[1+\left(f^{-m} u_{z}\right)_{z}\right]
\end{gathered}
$$

where the subscripts refer to partial derivatives, $k=k_{0} f^{n}$ and $\eta^{*}=\eta_{0} f^{-m}$ are assumed, and the units of length and time are $\left(\mathrm{k}_{0} \eta_{0} / \eta_{1}\right)^{1 / 2}$ and $\left(\eta_{0} \eta_{1} / \mathrm{k}_{0}\right)^{1 / 2}(\mathrm{~g} \Delta \rho)^{-1}$, respectively. The algebraic Darcy's law is recovered in the form $u=f^{n}$, if $u_{z}=0$. The second term on the RHS of equation (4) can be interpreted as the deviation of the liquid pressure gradient from the hydrostatic prediction which occurs because the deformation of the matrix sets up a pressure difference between the liquid and matrix.

Focussing on the problem of melt migration in the earth's mantle, there are major uncertainties in the choice of $\mathrm{k}_{0}, \mathrm{n}, \eta_{0}$ and $\mathrm{m}$. $\mathrm{k}_{0}$ depends roughly on the square of the spacing between liquid channels (i.e. the grain size); $n$ ranges from 2 to 5 or more depending on the channel geometry [Dullien, 1979]; $\eta_{0}$ may be much lower than the viscosity of the matrix phase if compaction is enhanced by the presence 


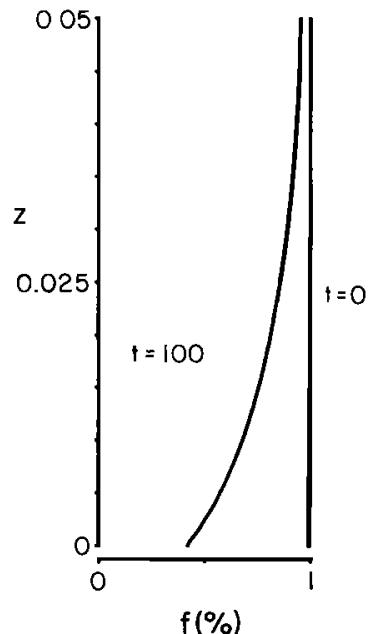

Fig. 1. A simple example illustrating the control exerted by matrix compaction on liquid ascent. The vertical scale is non-dimensional height, the horizontal scale is liquid fraction in percent. The plot shows the profile of liquid fraction, initially and after 100 time units. The initial state is a uniform liquid fraction of $1 \%$. The bottom boundary is impermeable $(u=0)$, so the matrix must compact in the lower region to allow liquid to escape. Simple analysis predicts the non-dimensional height of the compacting boundary layer to be $\mathrm{f}^{(\mathrm{n}-\mathrm{m}) / 2}$, where $\mathrm{n}$ and $\mathrm{m}$ are the permeability and effective viscosity parameters in equation (4). Here $n-3$ and $m=1$ so the height is 0.01 units. The layer develops in a non-dimensional time of $\mathrm{f}^{1-(\mathrm{n}+\mathrm{m}) / 2}$, here equal to 100 units. Liquid escapes freely through the top boundary, so the deviation from Darcy's law in the upper part of the column is small.

of the liquid; $m$ probably lies between 0 and 1 . The effective viscosity $\eta^{*}$ includes a bulk (or 'second') viscosity that has never been measured for a partially molten medium. However, recent experiments [Cooper and Kohlstedt, 1984] on an olivine-basalt assemblage indicate that the shear viscosity in the partially molten state is not greatly reduced from that in the completely solid state. We therefore feel justified in using a typical asthenospheric estimate, $\eta_{0} \approx \eta_{\mathrm{m}} \approx 10^{20}$ Poise. With a grain size of $1 \mathrm{~mm}$, the unit of length is then $\approx 50 \mathrm{~km}$ and the unit of time is $\approx 10,000 \mathrm{yr}$.

We have studied the system of equations (3) and (4) using numerical and analytic techniques, concentrating on the one-dimensional case and on understanding qualitatively the processes in igneous systems. A range of power laws have been used for the matrix permeability and effective viscosity $(\mathrm{n} \geqslant 2, \mathrm{~m}=0$ or 1$)$; these changes have surprisingly little qualitative effect on the solutions. The quantitative behaviour is, however, strongly affected, adding to the uncertainties in choosing the length and time units. For example, the height of a simple compacting boundary layer (the compaction length [McKenzie, 1984]), as shown in figure 1, depends on $\mathrm{n}$ and $\mathrm{m}$ as well as the liquid fraction and the length unit.

\section{Solitons}

We can demonstrate solitary wave solutions which propagate over a constant, low background liquid fraction $\mathrm{f}_{0}$. In the usual way [Drazin, 1983], these solutions are sought by substitution of $f=\varphi(y), y \equiv z-c t$, in equations (3) and (4); c is the ascent velocity of the wave. Integration of (4) gives $\mathrm{u}=\mathrm{u}_{0}+c \varphi$, where $\mathrm{u}_{0}$ is a constant of integration. As $\mathrm{y} \rightarrow \pm \infty$, in the background region, we demand $\varphi=f_{0}, \varphi_{y}=\varphi_{y y}=0$; hence $f_{0}{ }^{n}=u_{0}+c f_{0}$ and $u_{0}$ can be eliminated (again, the subscript $y$ denotes differentiation). The following ordinary differential equation in $\varphi$ is obtained from equation (4):

$$
\frac{\left[c\left(\varphi-f_{0}\right)-\left(\varphi^{n}-f_{0}^{n}\right)\right]}{\varphi^{n}}=c\left(\varphi^{-m^{n}} \varphi_{y}\right)_{y}
$$

which can be integrated once using the identity

$$
\left(\varphi^{-\mathrm{m}} \varphi_{\mathrm{y}}\right)_{\mathrm{y}} \equiv 1 / 2 \varphi^{\mathrm{m}} \frac{\mathrm{d}}{\mathrm{d} \varphi}\left[\varphi^{-2 \mathrm{~m}} \varphi_{\mathrm{y}}{ }^{2}\right]
$$

In the case $m=0$, we obtain (for $n \neq 2$ ),

$$
\begin{aligned}
\varphi_{y}= \pm\left(\frac{2 f_{0}}{c}\right)^{1 / 2} & {\left[\frac{c^{*}}{(n-1)(n-2)}-\psi+\right.} \\
& \left.\frac{n}{(n-1)}-\frac{c^{*}}{(n-2) \psi^{n-2}}+\frac{\left(c^{*}-1\right)}{(n-1) \psi^{n-1}}\right]^{1 / 2}
\end{aligned}
$$

where $c^{*}=c / f_{0}{ }^{n-1}$, and $\psi=\varphi / f_{0}$. $c^{*}$ and $\psi$ are the ascent velocity and profile of the wave scaled to the ascent velocity of the background liquid and the background liquid fraction. In the special case of $n=2$, the expression inside the square bracket is replaced by

$$
\left[c^{*}\left(\ln \psi-1+\frac{1}{\psi}\right)-\left(\psi-2+\frac{1}{\psi}\right)\right]
$$

Solitary wave solutions exist provided $\varphi_{\mathrm{y}}{ }^{2}$ is positive for some finite range of $\psi$ and goes to zero at $\psi=\Psi \neq 1$ as well as at $\psi=1 . \Psi$ is then the scaled wave amplitude. These requirements are satisfied for $\psi>1$ ('positive' solitary waves) provided $n>1$. The dispersion relation, obtained by setting $\varphi_{\mathrm{y}}=0$ at $\psi=\Psi$, is, for $\mathrm{n} \neq 2$;

$$
c^{*}=(n-1)(n-2) \frac{\left[\Psi-\frac{n}{(n-1)}+\frac{1}{(n-1) \Psi^{n-1}}\right]}{\left[1-\frac{(n-1)}{\Psi^{n-2}}+\frac{(n-2)}{\Psi^{n-1}}\right]}
$$

$$
\text { and for } \mathrm{n}=2 ; \quad \mathrm{c}^{*}=\frac{\left[\Psi-2+\frac{1}{\Psi}\right]}{\left[\ln \Psi-1+\frac{1}{\Psi}\right]}
$$

In the limit $\Psi \gg 1$, equation (6) can be integrated to give $\psi \approx \Psi-\mathrm{y}^{2} / 2 \mathrm{cf}_{0}, \psi \gg 1$, implying an approximately parabolic solitary wave.

In a similar way, the case $m=1$ leads to solitary waves for n $>1$, with approximately Gaussian form. The corresponding dispersion relation is:

$$
c^{*}=n(n-1) \frac{\left[\ln \Psi-\frac{1}{n}+\frac{1}{n \Psi^{n}}\right]}{\left[1-\frac{n}{\Psi^{n-1}}+\frac{(n-1)}{\Psi^{n}}\right]}
$$




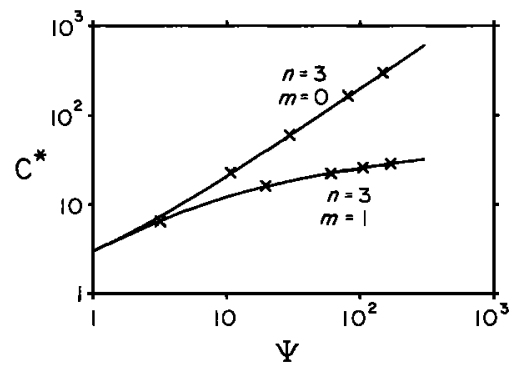

Fig. 2. Dispersion relations between the scaled ascent velocity $c^{*}$ and scaled amplitude $\Psi$ of solitary waves (see text). The solid lines are the theoretical predictions for two choices of $n$ and $\mathrm{m}$; the crosses are values measured in numerical experiments. These experiments used $\mathrm{f}_{0}=0.1 \%$, so values of $\Psi$ greater than about 300 (corresponding to a liquid fraction greater than $30 \%$ ) are physically unreasonable since the solid phase would no longer be connected (Arzi,1978).

For all $\mathrm{m}, \mathrm{c}^{*} \rightarrow \mathrm{n}$ as $\Psi \rightarrow 1$ (small amplitude waves), in agreement with the dispersion relation obtained by linearising equation (4). In analogy with the Korteweg-de Vries equation, it is found that the linearised frequency-wavenumber relation predicts dispersion but the non-linearity causes wave-steepening.

In numerical experiments, using an iterative finitedifference scheme, these solitary waves are found to be stable and arise whenever a substantially liquid region underlies a column of low liquid fraction. The liquid preferentially forms solitary waves which ascend through the column with

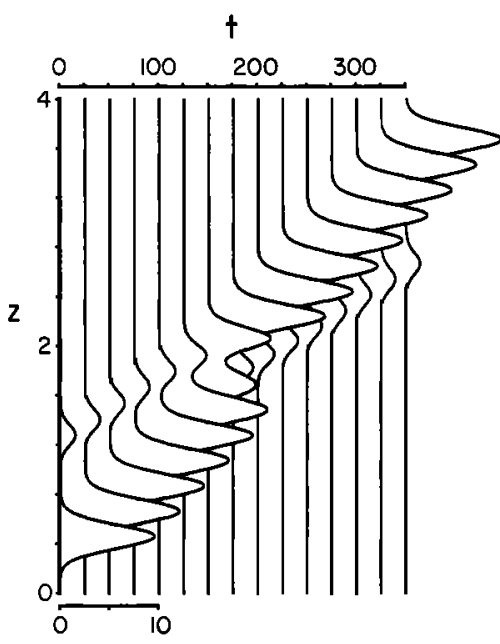

$f(\%)$

Fig. 3. The interaction of two solitons. Vertical profiles of liquid fraction are shown at successive points in nondimensional time, as indicated by the upper horizontal axis. In this calculation, we use $n=2$ in the matrix permeability and $m=1$ in the effective matrix viscosity. The initial state consists of two solitons (approximately Gaussian when $m=1$ ) in a column containing a uniform background liquid fraction of $0.1 \%$. The flux at the upper and lower boundaries is prescribed to maintain this background value. If alone, the individual solitons would ascend through the column at a constant velocity with unchanging form. Here, the larger soliton ascends faster due to its greater total buoyancy, and overtakes the smaller. Both emerge essentially unchanged by the interaction, apart from the phase shifts seen in the plot. The background liquid fraction is also unchanged. an unchanging profile, at a constant velocity. Figure 2 shows the exact dispersion relations derived above, for two choices of $\mathbf{n}$ and $\mathrm{m}$, and points from corresponding numerical experiments in which solitary wave amplitudes and velocities were measured. The agreement lends us confidence in the numerical technique. The sizes and shapes of the numerically created solitary waves are also in agreement with theoretical predictions.

In many non-linear systems exhibiting solitary waves [Fornberg and Whitham, 1978], the form and amplitude of the individual waves are conserved in collisions, either exactly or approximately. In these cases, the waves may be termed solitons. This type of interaction is shown in figure 3 , demonstrating that the solitary wave solutions to our equation are solitons. Figure 4 shows how the episodic ascent process can arise from a steady-state melting process, that might occur in a rising mantle plume. Referring to figures 3 and 4 , we again note the qualitative similarities but quantitative differences that arise from different choices of $\mathbf{n}$ and $\mathbf{m}$.

\section{Discussion}

When discussing consequences of magma solitons in the geological context, we propose the abbreviation 'magmon'. A resolution of the quantitative uncertainties described above is needed because the significance of magmons in igneous processes will depend on their physical size and velocity. This may come through experimental determination of the appropriate matrix permeability and effective viscosity. Plausible estimates of the various parameters lead to magmon heights of a few $\mathrm{km}$ and velocities of a few tens of $\mathrm{cm} / \mathrm{yr}$, in which case the geodynamic and geochemical consequences

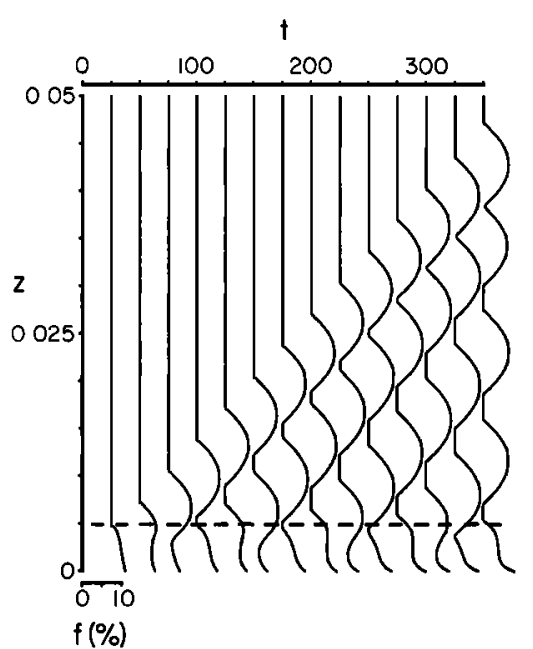

Fig. 4. Creation of solitons by a melting event. Layout of the plot is as in figure 3. This calculation uses an $n=3$ permeability (giving narrower solitons) and a constant $(\mathrm{m}=0)$ effective matrix viscosity (giving the approximately parabolic soliton profiles). Initially the column contains only a background liquid fraction of $0.1 \%$. Melting then occurs in the lowest tenth of the column, below the dashed horizontal line, and continues at a constant rate. Based on more complete calculations that include melting and thermal effects, this is a reasonable simulation of the pressure-release melting of a rock that consists of a low-melting fraction and a refractory residue. Solitons rise individually, separated by necks where the liquid fraction returns to its background value. Note that the first soliton has a slightly lower amplitude and velocity than the second. The latter catches up and in the last few profiles the type of interaction shown in figure 3 is occurring. 
may be resolvable in observations. For example, the episodic ascent process shown in figure 4 would lead to temporally, and perhaps spatially, periodic eruptions. Within a magmon, the liquid is in intimate contact with the matrix, and will acquire some chemical imprint as it ascends. The existence of magmons is also conditional on their stability in two and three spatial dimensions, which is the subject of continuing study. Even if instability occurs (for example, self-focussing [Kelley, 1965; Kadomstev and Petviashvili, 1970]) occurs, it may have interesting consequences such as streamers or plumes of rising liquid. The possibility of solitary waves in other porous flow problems also deserves attention.

Acknowledgements. We are grateful to numerous colleagues for comments and suggestions, in particular $D$. Anderson, R. Clayton, B. Hager, B. Kamb, O. Navon, E. Stolper, D. Turcotte and G. Whitham. Frank Richter and Dan McKenzie are conducting similar research and have generously shared their results with us. Supported in part by NSF Grant EAR-8206383. Contribution number 4102, Division of Geological and Planetary Science, California Institute of Technology, Pasadena, CA 91125.

\section{References}

Arzi, A. A., Critical phenomena in the rheology of partially molten rocks, Tectonophysics, 44, 173-184, 1978.

Bear, J., Dynamics of Fluids in Porous Media, 764pp., American Elsevier, New York, 1972.

Cooper, R. F. and D. L. Kohlstedt, Solution-precipitation enhanced diffusional creep of partially molten olivinebasalt aggregates, Trans. Am. Geophys. Un. EOS, 65, 280, 1984.

Drazin, P. G., Solitons, 136pp., London Mathematical Society Lecture Notes Series 85, Cambridge University Press, 1983.
Dullien, F. A. L., Porous Media Fluid Transport and Pore Structure, Academic Press, New York, 1979.

Fornberg, B. and G. B. Whitham, A numerical and theoretical study of certain non-linear wave phenomena, Phil. Trans. R. Soc. London, 289, 373-404, 1978.

Fowler, A. C., On the transport of moisture in polythermal glaciers, Geophys. Astrophys. Fluid Dynamics, 28, 99-140, 1984.

Kadomstev, B. B. and V. I. Petviashvili, On the stability of solitary waves in weakly dispersing media, Sov. Phys. Dokl., 15, 539-541, 1970.

Kelley, P. L., Self-focusing of optical beams, Phys. Rev. Lett., 15, 1005-1008, 1965.

McKenzie, D. P., The generation and compaction of partial melts, J. Petrology, in press, 1984.

Nye, J. F., The flow law of ice from measurements in glacier tunnels, laboratory experiments and the Jungfraufirn borehole experiment, Proc. R. Soc. London, A219, 477$489,1953$.

Sleep, N. H., Segregation of a magma from a mostly crystalline mush, Geol. Soc. Am. Bull., 85, 1225-1232, 1974.

Stevenson, D. J., Self regulation and melt migration (can magma oceans exist?), Trans. Am. Geophys. Un. EOS, 61, $1021,1980$.

Turcotte, D. L., Magma migration, Ann. Rev. Earth Planet. Sci., 10, 397-408, 1982.

David R. Scott and David J. Stevenson, Division of Geological and Planetary Science, California Institute of Technology, Pasadena, CA 91106.

(Received August 31, 1984; accepted September 25, 1984.) 